\title{
Chewing gum promotes bowel function recovery in elderly patients after lumbar spinal surgery: a retrospective single-center cohort study
}

\author{
Xing Du, Yunsheng Ou, Guanyin Jiang, Wei Luo, Dianming Jiang \\ Department of Orthopedics, The First Affiliated Hospital of Chongqing Medical University, Chongqing, China \\ Contributions: (I) Conception and design: X Du, Y Ou; (II) Administrative support: Y Ou; (III) Provision of study materials or patients: Y Ou; (IV) \\ Collection and assembly of data: X Du, G Jiang; (V) Data analysis and interpretation: X Du, W Luo, D Jiang; (VI) Manuscript writing: All authors; \\ (VII) Final approval of manuscript: All authors. \\ Correspondence to: Yunsheng Ou. Department of Orthopedics, The First Affiliated Hospital of Chongqing Medical University, No. 1 YouYi Road, \\ Yuan Jia Gang, Yu Zhong District, Chongqing 400016, China. Email: ouyunsheng2001@163.com.
}

\begin{abstract}
Background Postoperative paralytic ileus is not a rare complication after lumbar spinal surgery especially in elderly patients. Chewing is a kind of sham feeding that has been reported to stimulate bowel motility, but so far there was no study showed these positive results may or may not be extrapolated to the spinal surgery population. We sought to determine whether chewing gum facilitates bowel function recovery in elderly patients undergoing lumbar spine surgery.

Methods: Sixty consecutive elderly patients (over 60 years old) with lumbar degenerative diseases undergoing posterior lumbar fusion surgery between September 2017 and April 2019 were respectively included and divided into two groups. The chewing gum group (30 patients) started chewing gum after they were awakened from anesthesia until the first defecation occurred, while the control group (30 patients) chewed nothing. The time to first flatus, first bowel sounds heard and first defecation, the length of hospital stay and postoperative complications were all recorded and analyzed.
\end{abstract}

Results: Compared with control group, the chewing gum group had less time to the first flatus $(12.4 \pm 2.9$ vs. 17.8 $\pm 2.2 \mathrm{~h} ; \mathrm{P}<0.001)$, first bowel sounds heard $(17.3 \pm 2.8$ vs. $25.0 \pm 2.5 \mathrm{~h} ; \mathrm{P}<0.001)$ and first defecation (51.9 \pm 5.2 vs. $76.1 \pm 3.8 \mathrm{~h} ; \mathrm{P}<0.001)$, but no significant differences were found in the length of hospital stay $(11.7 \pm 2.1$ vs. $11.9 \pm 2.5 \mathrm{~d} ; \mathrm{P}=0.697)$ and the postoperative complications $(\mathrm{P}=0.501)$.

Conclusions: This study demonstrated that chewing gum can promote bowel function recovery in elderly patients after lumbar spinal surgery.

Keywords: Elderly; chewing gum; postoperative bowel function recovery; lumbar spinal surgery

Submitted May 06, 2020. Accepted for publication Sep 02, 2020.

doi: 10.21037/apm-20-1077

View this article at: http://dx.doi.org/10.21037/apm-20-1077

\section{Introduction}

Postoperative paralytic ileus is defined as temporary gastrointestinal motility dysfunction after surgery, which is characterized by abdominal pain/distention, belching, nausea, vomiting and constipation (1). It may lead to increased postoperative pain, patients' discomfort, delayed enteral nutrition, poor wound healing, delayed mobilization and increased health care costs (2). Although most of postoperative paralytic ileus occurred in patients after abdominal surgery, it is not rarely reported in orthopedic surgery $(3,4)$. Postoperative paralytic ileus is reported as a frequent complication after lumbar spinal surgery, occurring in $2.6 \%$ to $12 \%$ depending on the invasiveness and approach of the surgery (5). Moreover, China is facing a rapid aging population (6), and lumbar spinal surgery in the aging population is also reported being on 
the rise (7). Elderly patients were often complicated with chronic constipation (8), and thus may suffer higher risk of postoperative ileus after orthopedic surgery than the younger (9). Therefore, it is of great significance to find effective measures to prevent postoperative paralytic ileus in the elderly after lumbar spinal surgery.

To prevent postoperative paralytic ileus, many therapies including early enteral nutrition, early removal of nasogastric tube, gastrointestinal motility drugs and physical therapy have been put forward and applied in clinical work (10-12). However, they can not be routinely or widely used because of the low compliance of the former two and limited clinical efficacy of the latter two $(13,14)$. It has been reported that chewing gum can promote gastrointestinal motility after abdominal surgery such as caesarean section and colorectal resection (15-17), but so far there was no study showed these positive results may or may not be extrapolated to the orthopedic surgery population.

Therefore, we conducted this retrospective singlecenter cohort study to investigate the influence of chewing gum on gastrointestinal motility function recovery in elderly patients after lumbar spinal surgery. We present the following article in accordance with the STROBE reporting checklist (18). Available at http://dx.doi.org/10.21037/apm20-1077.

\section{Methods}

This retrospective single-center cohort study was conducted in accordance with the Declaration of Helsinki (as revised in 2013). The study was approved by Ethics Committee of the First Affiliated Hospital of Chongqing Medical University (No.: 2018-089) and all of the participants provided their written informed consent before their data were recorded and stored.

\section{Patients selection}

Consecutive patients undergoing posterior lumbar fusion surgery for degenerative lumbar diseases under general anesthesia at our department between September 2017 and April 2019 were respectively selected.

The inclusion criteria: (I) patients who were diagnosed with degenerative lumbar diseases, such as lumbar disc herniation (LDH), lumbar spinal stenosis (LSS) or lumbar spondylolisthesis (LS), based on clinical symptoms (e.g., low back pain, lower limb pain or numbness) and radiological imaging; (II) patients who were over 60 years old; (III) patients undergoing conservative therapy for at least 3 months without improvement; (IV) patients who received open posterior lumbar fusion surgery, such as posterior lumbar interbody fusion (PLIF) or transforaminal lumbar interbody fusion (TLIF), under general anesthesia.

The exclusion criteria: (I) patients with a history of spinal or gastrointestinal surgery; (II) patients who can not understand the experiment content or unable to cooperate (e.g., neural mental diseases, poor education level); (III) patients with high risk of esophageal obstruction (e.g., dentures, loose teeth); (IV) patients with spinal fractures, tumor, infection, or spinal deformity; (V) patients with diabetes mellitus, gastrointestinal dysfunction, or severe cerebrovascular diseases.

\section{Postoperative management}

All the surgical procedures (PLIF or TLIF) were performed by a senior spinal surgeon according to the standard operation method. The surgery was done in the prone position under general anesthesia. A midline skin incision was made to expose the spinous processes, lamina, facets, and transverse processes of the involved lumbar levels. The pedicle screws were implanted with the C-arm radiography confirmation. Total laminectomy or hemilaminectomy was performed to decompress the nerve root or spinal canal. Then, discectomy was done and a cage filled with the autograft was implanted for interbody fusion. Finally, the operation field was flushed by normal saline and the incision was closed layer by layer after placing a drainage tube. After operation, the chewing gum group started chewing gum 4 hours after they were totally awaken of anesthesia. They chewed gum every 4 hours, 4 grain every time and at least for 15 minutes. When first passage of flatus occurred, the gum chewing frequency was decreased to every 8 hours until first defecation. A commercially available sugarless gum (Extra, Wm Wrigley Jr Company, China agent) was used. The control group did not chew gum. Standard postoperative care interventions (e.g., prophylactic antibiotics, dehydrating agents) were provided for all patients. Patientcontrolled analgesia (PCA) was only used in the patients who signed the consent for use preoperatively. When first bowel sounds were heard, patients were allowed to start oral intake of liquid, semi-fluid or soft foods, and then gradually transition to a normal diet.

\section{Measurement}

Preoperative demographic data [e.g., age, gender, body mass 
index (BMI), diagnosis, comorbidities] and surgery related variables (e.g., ASA grade, type of operation, operative level, operative duration and blood loss, the use of postoperative PCA) were all recorded. We defined severe constipation as follows: (I) onset of constipation symptoms at least 6 months prior to spinal surgery; (II) previous treatment with at least 2 laxatives from different classes for at least 6 months; (III) no evidence of organic diseases causing constipation. Patients who meet the above three points are considered to be serious constipation. Preoperative opioid medication of the patients was also collected and compared. The time of the first gum chewing was defined as the 0 hour. Postoperative bowel sounds of each patient was checked by two research members every half an hour using a standard stethoscope. All patients were asked to notify the nurse or medical staff when first passage of flatus and the first defecation occurred. The length of hospital stay was defined as the period from patient's admission to discharge. The postoperative complications included esophageal obstruction caused by gum, vomiting, abdominal distension, intestinal obstruction, urinary retention, pulmonary infection and wound infection. Clavien-Dindo classification was used to evaluate the postoperative complications (19).

\section{Statistical analysis}

Quantitative data was represented as mean \pm standard deviation and compared by $t$-test. Qualitative data was recorded as number (percentages). Disordered qualitative data (e.g., gender) was compared by Chi square test or Fisher's exact test and ordered qualitative data (e.g., ASA grade and Clavien-Dindo classification) was compared by Mann-Whitney U test. SPSS 22.0 software was used for statistical analysis and $\mathrm{P}<0.05$ was considered to be the significant difference.

\section{Results}

A total of 60 patients were finally included in this study, involving 30 cases in the chewing gum group and 30 cases in the control group. There was no significant difference in age, gender, BMI, preoperative diagnosis, comorbidities and serious constipation between the two groups (Table 1).

No significant differences were found in the ASA grade, type of operation, operative level and postoperative PCA use. The operative duration and operative blood loss in the two groups were $139.5 \pm 27.0,138.7 \pm 34.5 \mathrm{~min}$ and $156.7 \pm 76.3182 .3 \pm 154.9 \mathrm{~mL}$, respectively $(\mathrm{P}>0.05)$. The comparisons of surgery related variables between the two groups were shown in Table 2.

We found a significant shorter time to first flatus in chewing gum group compared with control group $(12.4 \pm 2.9$ vs. $17.8 \pm 2.2 \mathrm{~h} ; \mathrm{P}<0.001)$. The time to first bowel sounds heard $(17.3 \pm 2.8$ vs. $25.0 \pm 2.5 \mathrm{~h} ; \mathrm{P}<0.001)$ and first defecation $(51.9 \pm 5.2$ vs. $76.1 \pm 3.8 \mathrm{~h} ; \mathrm{P}<0.001)$ were both significantly reduced in chewing gum group compared with control group. However, no significant difference was found in length of hospital stay $(11.7 \pm 2.1 v s .11 .9 \pm 2.5 \mathrm{~d}$; $\mathrm{P}=0.697)$. The comparisons of postoperative clinical outcomes between the two groups were shown in Table 3.

According to the Clavien-Dindo classification, there were 7 case of complications in the chewing gum group, including 2 cases of grade I ( 1 case of vomiting, 1 case of abdominal distension), 5 cases of grade II (1 case of vomiting, 1 case of abdominal distension, 2 cases of urinary retention and 1 case of pulmonary infection). In the control group, there were 10 cases of complications, including 2 cases of grade I ( 1 case of vomiting, 1 case of abdominal distension), 7 cases of grade II ( 2 cases of vomiting, 1 case of abdominal distension, 1 case of non-symptomatic intestinal obstruction, 2 cases of urinary retention and 1 case of pulmonary infection), and 1 case of grade III (1 case of wound infection). No significant difference was found in postoperative complications between the two groups $(\mathrm{P}=0.501)$. The comparison of postoperative complications between the two groups was shown in Table 4. All the complications were recovered after active treatment.

\section{Discussion}

It is reported that the mean incidence of postoperative ileus after lumbar surgery is about $3.5 \%(20,21)$, and it varies with the surgical approach. Fineberg et al. conducted a retrospective study involving more than 200,000 patients and found that a posterior lumbar spinal fusion surgery had a mean postoperative ileus rate of $2.6 \%$ compared with $7.5 \%$ for an anterior approach and the incidence can be as high as $12 \%$ for a combined anterior and posterior approach (5). Moreover, an increasing trend for lumbar spinal surgery in Chinese elderly patients was also widely reported because of the aging of the population $(6,7)$. As most of the elderly suffer chronic constipation (8), the risk of postoperative ileus in elderly patients may be higher than that previously reported in the younger $(9,22)$. Thus, although paralytic ileus is considered a mild complication of spinal surgery in previous study (23), it occurs more frequently than we 
Table 1 Comparison of preoperative demographic data between the two groups

\begin{tabular}{|c|c|c|c|}
\hline Preoperative demographic data & Control group $(\mathrm{n}=30)$ & Chewing gum group $(n=30)$ & $P$ value \\
\hline Gender & & & 0.602 \\
\hline Male & 18 & 16 & \\
\hline Female & 12 & 14 & \\
\hline Serious constipation (n) & 3 & 5 & 0.706 \\
\hline Spinal disease (n) & & & 0.690 \\
\hline LDH & 9 & 10 & \\
\hline LSS & 17 & 14 & \\
\hline Hypertension & 3 & 5 & \\
\hline Diabetes & 4 & 3 & \\
\hline Cardiovascular diseases & 3 & 2 & \\
\hline Lung diseases & 1 & 2 & \\
\hline Preoperative opioid medication (n) & 5 & 7 & 0.519 \\
\hline
\end{tabular}

LDH, lumbar disc herniation; LSS, lumbar spinal stenosis; LS, lumbar spondylolisthesis; BMI, body mass index.

Table 2 Comparison of surgery related variables between the two groups

\begin{tabular}{|c|c|c|c|}
\hline Surgery related variables & Control group $(n=30)$ & Chewing gum group $(n=30)$ & $P$ value \\
\hline I & 19 & 17 & \\
\hline II & 9 & 11 & \\
\hline III & 2 & 2 & \\
\hline PLIF & 8 & 10 & \\
\hline TLIF & 22 & 20 & \\
\hline Operative levels $(n)$, mean $\pm S D$ & & & 0.432 \\
\hline 1 & 19 & 16 & \\
\hline Blood loss $(\mathrm{mL})$, mean $\pm \mathrm{SD}$ & $156.7 \pm 76.3$ & $182.3 \pm 154.9$ & 0.419 \\
\hline Postoperative PCA & 22 & 26 & 0.197 \\
\hline
\end{tabular}

PLIF, posterior lumbar interbody fusion; TLIF, transforaminal lumbar interbody fusion; PCA, patient-controlled analgesia. 
Table 3 Comparison of postoperative clinical outcomes between the two groups

\begin{tabular}{|c|c|c|c|}
\hline Clinical outcomes & Control group $(n=30)$ & Chewing gum group $(n=30)$ & $P$ value \\
\hline Time to first bowel sounds (h) & $17.8 \pm 2.2$ & $12.4 \pm 2.9$ & $<0.001$ \\
\hline Time to first defecation (h) & $76.1 \pm 3.8$ & $51.9 \pm 5.2$ & $<0.001$ \\
\hline Length of hospital stay (d) & $11.9 \pm 2.5$ & $11.7 \pm 2.1$ & 0.697 \\
\hline
\end{tabular}

Table 4 Comparison of postoperative complications between the two groups

\begin{tabular}{lcc}
\hline Complications (Clavien-Dindo classification) & Control group $(\mathrm{n}=30)$ & Chewing gum group $(\mathrm{n}=30)$ \\
\hline II & 2 & 2 \\
II & 7 & 5 \\
III & 1 & 0 \\
In total & 10 & $7^{\#}$ \\
\hline
\end{tabular}

\#, Mann-Whitney U test was performed, $\mathrm{P}=0.501$, compared with the control group.

ever thought, especially in elderly patients. Therefore, it is of importance to find out proper management to promote bowel function recovery in elderly patients after spinal surgery.

At present, treatment of postoperative ileus mainly includes perioperative prevention, traditional treatment, pharmacologic interventions and surgical treatment (24). Traditional treatment such as nasogastric decompression, electrolyte replacement and early ambulation have been reported with limited clinical efficacy and poor patient compliance (25). Pharmacologic interventions are commonly used following abdominal surgery to prevent ileus, such as prokinetic motility drugs, antiemetics, neostigmine and $\mu$-receptor antagonist, but they had been found of little or even no benefit (26,27). Surgery is only indicated for the serious complications due to intestinal obstruction, such as bowel perforation or ischemia. So, the postoperative prevention plays an important role in the management of postoperative ileus.

Chewing is a kind of sham feeding that has been reported to stimulate bowel motility in humans (28). The possible physiological mechanisms are as follows: (I) chewing gum can stimulate the chemical and mechanical receptors of oropharynx, activate the cephalic-vagal nerve pathway, and increase the secretion of gastrointestinal hormones such as gastric acid, pepsinogen, gastrin and motilin, thus promoting the motility of gastrointestinal tract $(29,30)$; (II) masticatory action itself can also stimulate the vagus nerve pathway, increase the release of acetylcholine transmitters; the acetylcholine transmitters bind to nicotine receptors on inflammatory cells, thus reducing the release of proinflammatory factors and promoting the recovery of gastrointestinal motility (31); (III) some studies have shown that xylitol contained in sugar-free gum had the effect of affecting gastrointestinal motility and mild permeable diarrhea, which may also be the reason why chewing gum promotes the recovery of intestinal paralysis (32). Therefore, chewing gum can promote gastrointestinal motility mainly by directly activating cephalic-vagal nerve pathway and indirectly promoting the secretion of gastrointestinal hormones.

In our study, the results showed that chewing gum can promote bowel function recovery in elderly patients after posterior lumbar surgery. The time to first flatus, first bowel sounds heard and first defecation were all significantly accelerated. The bowel motility was significantly accelerated in both two groups compared with that reported in abdominal surgery ( 3 to 4 days). There are several potential explanations. Firstly, the operative duration of posterior lumbar fusion surgery was usually very short (less than 3 hours). Secondly, there is less intestinal inflammatory response during the posterior lumbar fusion surgery that reduce the risk of postoperative ileus after posterior lumbar surgery compared with abdominal surgery. Thirdly, in 
posterior lumbar surgery, however, the bowel is hardly intervened.

A shorter hospitalization time in the chewing gum group was expected based on its promoting bowel motility recovery, but our data did not demonstrate a notable difference, this result was different with the previous metaanalyses $(33,34)$. There may be the following reasons: (I) the sample size of this study is small with only 60 cases included; (II) the hospitalization time was related variable factors, such as the time of preoperative examination, the change of patient's health condition, or even the insurance system which covers most of the hospitalization fees; (III) surgeons may have different experiences in the indication of discharge. Moreover, no serious complications were found in the two groups indicating the tolerance and safety of chewing gum in elderly patients.

This study also had some limitations. Firstly, it was a single center retrospective study with small sample size. Secondly, research members may have different experiences in the auscultation of the bowel sounds and thus may cause some heterogenicity. Thirdly, the time to first passage of flatus and first defecation were provided by the patients or their family members, and there may be a lack of inaccuracy or timeliness.

\section{Conclusions}

Postoperative chewing gum enhances the bowel function recovery in elderly patient after lumbar spine surgery without serious complications, and thus it might be recommended in clinical work. However, since this was a small-sample retrospective study, prospective randomized control studies with large sample size are needed to validate our findings.

\section{Acknowledgments}

The authors would like to thank Yong Zhu, Zenghui Zhao, Jianxiao Li and Wei Zhang for the technical assistant.

Funding: This study was supported by the Natural Science Foundation of Chongqing (No. cstc2019jcyj-msxmX0358).

\section{Footnote}

Reporting Checklist: The authors have completed the STROBE reporting checklist. Available at http://dx.doi. org/10.21037/apm-20-1077
Data Sharing Statement: Available at http://dx.doi. org/10.21037/apm-20-1077

Conflicts of Interest: All authors have completed the ICMJE uniform disclosure form (available at http://dx.doi. org/10.21037/apm-20-1077). The authors have no conflicts of interest to declare.

Etbical Statement: The authors are accountable for all aspects of the work in ensuring that questions related to the accuracy or integrity of any part of the work are appropriately investigated and resolved. The study was conducted in accordance with the Declaration of Helsinki (as revised in 2013). The study was approved by Ethics Committee of the First Affiliated Hospital of Chongqing Medical University (No.: 2018-089) and individual consent for this retrospective analysis was also obtained from the patient.

Open Access Statement: This is an Open Access article distributed in accordance with the Creative Commons Attribution-NonCommercial-NoDerivs 4.0 International License (CC BY-NC-ND 4.0), which permits the noncommercial replication and distribution of the article with the strict proviso that no changes or edits are made and the original work is properly cited (including links to both the formal publication through the relevant DOI and the license). See: https://creativecommons.org/licenses/by-nc-nd/4.0/.

\section{References}

1. Vather R, Trivedi S, Bissett I. Defining postoperative ileus: results of a systematic review and global survey. J Gastrointest Surg 2013;17:962-72.

2. Doorly MG, Senagore AJ. Pathogenesis and clinical and economic consequences of postoperative ileus. Surg Clin North Am 2012;92:259-72.

3. Venara A, Neunlist M, Slim K, et al. Postoperative ileus: Pathophysiology, incidence, and prevention. J Visc Surg 2016;153:439-46.

4. Daniels AH, Ritterman SA, Rubin LE. Paralytic ileus in the orthopaedic patient. J Am Acad Orthop Surg 2015;23:365-72.

5. Fineberg SJ, Nandyala SV, Kurd MF, et al. Incidence and risk factors for postoperative ileus following anterior, posterior, and circumferential lumbar fusion. Spine J 2014;14:1680-5. 
6. Cai J, Zhao H, Coyte PC. Socioeconomic Differences and Trends in the Place of Death among Elderly People in China. Int J Environ Res Public Health 2017;14:E1210.

7. Li Y, Zheng S, Wu Y, et al. Trends of surgical treatment for spinal degenerative disease in China: a cohort of 37,897 inpatients from 2003 to 2016. Clin Interv Aging 2019;14:361-6.

8. Vazquez Roque M, Bouras EP. Epidemiology and management of chronic constipation in elderly patients. Clin Interv Aging 2015;10:919-30.

9. Lee TH, Lee JS, Hong SJ, et al. Risk factors for postoperative ileus following orthopedic surgery: the role of chronic constipation. J Neurogastroenterol Motil 2015;21:121-5.

10. Toyomasu Y, Mochiki E, Morita H, et al. Mosapride citrate improves postoperative ileus of patients with colectomy. J Gastrointest Surg 2011;15:1361-7.

11. Adamakis I, Tyritzis SI, Koutalellis G, et al. Early removal of nasogastric tube is beneficial for patients undergoing radical cystectomy with urinary diversion. Int Braz J Urol 2011;37:42-8.

12. Ng SS, Leung WW, Mak TW, et al. Electroacupuncture reduces duration of postoperative ileus after laparoscopic surgery for colorectal cancer. Gastroenterology 2013;144:307-13.e1.

13. Saclarides TJ. Current choices -good or bad-for the proactive management of postoperative ileus: A surgeon's view. J Perianesth Nurs 2006;21:S7-S15.

14. Vather R, Bissett I. Management of prolonged postoperative ileus: Evidence based recommendations. ANZ J Surg 2013;83:319-24.

15. Vergara-Fernandez O, Gonzalez-Vargas AP, CastellanosJuarez JC, et al. Usefulness of Gum Chewing to Decrease Postoperative Ileus in Colorectal Surgery with Primary Anastomosis: A Randomized Controlled Trial. Rev Invest Clin 2016;68:314-8.

16. Wen Z, Shen $M, W u ~ C$, et al. Chewing gum for intestinal function recovery after caesarean section: a systematic review and meta-analysis. BMC Pregnancy Childbirth 2017;17:105.

17. Atkinson C, Penfold CM, Ness AR, et al. Randomized clinical trial of postoperative chewing gum versus standard care after colorectal resection. Br J Surg 2016;103:962-70.

18. von Elm E, Altman DG, Egger M, et al. The Strengthening the Reporting of Observational Studies in Epidemiology (STROBE) statement: guidelines for reporting observational studies. PLoS Med 2007;4:e296.

19. Clavien PA, Barkun J, de Oliveira ML, et al. The Clavien-
Dindo classification of surgical complications: five-year experience. Ann Surg 2009;250:187-96.

20. Kiely PD, Mount LE, Du JY, et al. The incidence and risk factors for post-operative ileus after spinal fusion surgery: a multivariate analysis. Int Orthop 2016;40:1067-74.

21. Deng WW, Lan M, Peng AF, et al. The risk factors for postoperative ileus following posterior thoracolumbar spinal fusion surgery. Clin Neurol Neurosurg 2019;184:105411.

22. Horowitz JA, Jain A, Puvanesarajah V, et al. Risk Factors, Additional Length of Stay, and Cost Associated with Postoperative Ileus Following Anterior Lumbar Interbody Fusion in Elderly Patients. World Neurosurg 2018;115:e185-9.

23. Caner H, Bavbek M, Albayrak A, et al. Ogilvie's syndrome as a rare complication of lumbar disc surgery. Can J Neurol Sci 2000;27:77-8.

24. ThompsonM, MagnusonB. Management of postoperative ileus. Orthopedics 2012;35:213-7.

25. Jain A, Vargas HD. Advances and challenges in the management of acute colonic pseudoobstruction(ogilviesyndrome). Clin Colon Rectal Surg 2012;25:37-45.

26. Kraft MD. Emerging pharmacologic options for treating postoperative ileus. Am J Health Syst Pharm 2007;64:S13-S20.

27. Oh CH, Ji GY, Yoon SH, et al. Paralytic Ileus and Prophylactic Gastrointestinal Motility Medication after Spinal Operation. Yonsei Med J 2015;56:1627-31.

28. Ho YM, Smith SR, Pockney P, et al. A meta-analysis on the effect of sham feeding following colectomy: should gum chewing be included in enhanced recovery after surgery protocols? Dis Colon Rectum 2014;57:115-26.

29. Stern RM, Crawford HE, Stewart WR, et al. Sham feeding. Cephalic-vagal influences on gastric myoelectric activity. Dig Dis Sci 1989;34:521-7.

30. Lunding JA, Nordström LM, Haukelid AO, et al. Vagal activation by sham feeding improves gastric motility in functional dyspepsia. Neurogastroenterol Motil 2008;20:618-24.

31. Mennella I, Ferracane R, Zucco F, et al. Food Liking Enhances the Plasma Response of 2-Arachidonoylglycerol and of Pancreatic Polypeptide upon Modified Sham Feeding in Humans. J Nutr 2015;145:2169-75.

32. Tandeter H. Hypothesis: hexitols in chewing gum may play a role in reducing postoperative ileus. Med Hypotheses 2009;72:39-40.

33. Xu C, Peng J, Liu S, et al. Effect of chewing gum on 
gastrointestinal function after gynecological surgery: A systematic literature review and meta-analysis. J Obstet Gynaecol Res 2018;44:936-43.

34. Liu Q, Jiang H, Xu D, et al. Effect of gum chewing on

Cite this article as: Du X, Ou Y, Jiang G, Luo W, Jiang D. Chewing gum promotes bowel function recovery in elderly patients after lumbar spinal surgery: a retrospective singlecenter cohort study. Ann Palliat Med 2021;10(2):1216-1223. doi: 10.21037/apm-20-1077 ameliorating ileus following colorectal surgery: A metaanalysis of 18 randomized controlled trials. Int J Surg 2017;47:107-15. 